\title{
Indoor Air Quality and Wellbeing - Enabling Awareness and Sensitivity with Ambient IoT Displays
}

\author{
Andreas Seiderer, Ilhan Aslan, Chi Tai Dang, and Elisabeth André \\ Augsburg University, Universitätsstr. 6a, 86159 Augsburg, Germany \\ \{seiderer, aslan\}@hcm-lab.de, \{dang, andre\}@informatik. uni-augsburg.de
}

\begin{abstract}
The quality of indoor air exerts influence on the wellbeing of people. However, people rarely notice a constant and creeping deterioration of indoor air. Especially in enclosed places where several people get together, like meeting rooms, school rooms and public transportation, bad air quality might cause a reduction of cognitive performance, increased headache, fatigue and sleepiness. This paper describes and discusses a privacy respecting system, built with low cost IoT components and open-source software, that informs room occupants about bad air quality with ambient lights. Additionally, the status of the windows is indicated so that they are not forgotten to be closed after airing. We conducted a workshop with an implementation of the system with five users and present the results which show the usefulness and desirability of ambient notifications for indoor air quality monitoring.
\end{abstract}

Keywords: Ambient display · Ambient light $\cdot$ Air quality $\cdot$ Smart home · Context-aware system · Human wellbeing · Ubiquitous computing.

\section{Introduction}

The indoor room climate plays an important role for human wellbeing, especially as humans do not directly and consciously perceive gases such as carbon dioxide $\left(\mathrm{CO}_{2}\right)$. It is therefore worrying that higher concentrations of $\mathrm{CO}_{2}$ can reduce perceived air quality, potentially affect cognitive performance $[13,11,36,33]$, or cause physiological symptoms $[13,26]$. Even perceivable gases are problematic since room occupants usually start to adapt to odors that slowly occur [20]. Such odors usually belong to volatile organic compounds (VOCs) which include gases emitted by humans (bioeffluents) but can for example also stem from cleaning agents. When entering a room, such odors are usually noticed and perceived as disturbing, whereby certain VOCs can also be harmful at higher concentrations.

Current sensor technologies can detect $\mathrm{CO}_{2}$ and VOCs, allowing to detect when a room should be ventilated in order to create a healthier environment. Buildings that have ventilation/air conditioning systems could automatically control the airflow in the building to reduce such gases. However, this is not standard in buildings and cannot simply be retrofitted. It therefore makes sense 
to notify people in a room about poor air quality in a non-disturbing way. Unobtrusive notifications can be achieved with ambient displays at the periphery of a room that are visible to all persons.

For this purpose, we developed a prototype that makes use of ambient lights to inform people in a meeting room about air quality and open windows. The ambient lights, located near each window handle and next to the door, encode air quality with the well-known colors of traffic lights. Furthermore, a slowly pulsed light pattern indicates that a window is still open. The pulse pattern occurs on the light at the door together with the lights at the open window(s) to prevent forgetting open windows when leaving the room. To make the lights less obtrusive the maximum brightness is adapted to the current lighting condition in the room. In addition, the lights are switched on only if there are room occupants in order to save energy.

Our prototype features privacy, flexibility, and reproducibility at low costs. The costs of the presented setup are ca. $440 €$ excluding the WiFi router (often already available), the SCD30, BMP280 and iAM sensors and the Arduino (just required for sensor comparison). High level of privacy is achieved by the sensors chosen and by processing and storing data only locally. It is not possible and not intended to determine data of an individual and a connection to the internet or a cloud is not required even though research has shown that cloud usage in such contexts might be accepted [16]. The system's hardware components are inexpensive, makes use of open-source software, and can be installed in any room by means of wireless smart home sensors and custom-built WiFi controlled RGB-LED actuators in addition to gas sensors connected to a Raspberry Pi.

In this paper we sum up related work and the resulting design decisions for our prototype and describe its implementation and installation in a meeting room of a lab at a university. We present insights on the data of multiple sensors that can be measured in this room. Furthermore, we report and discuss the results of a workshop conducted with five staff members that are using the room regularly. The descriptions and results of this paper can be used to implement comparable low-cost systems that could be installed in every room enabling room occupants to detect imperceptible gases and prevent a reduction of their wellbeing.

\section{Related Work}

\subsection{Influence of $\mathrm{CO}_{2}$ and Bioeffluents on Wellbeing and Cognitive Performance}

In order to determine color encodings for different air quality measures we reviewed literature for reasonable thresholds. The room in which our system is installed in is used for meetings where it is usually required that all persons are highly concentrated. Thus, we are especially interested in literature giving hints about thresholds where already slight reductions of the cognitive performance or wellbeing could be found.

We focus the discussion on $\mathrm{CO}_{2}$ and neglect VOC concentrations for the following reason. The low-cost VOCs sensor that we included has to be manually 
calibrated against a reference of "good" air quality (well aired room). In turn, the absolute readings of the sensor cannot be reliably used for comparison with values found in literature as the same value may refer to different qualities depending on the calibration. Nevertheless, this sensor still can provide helpful hints about odors in the room for data analysis.

A recent literature survey by Azuma et al. [13] gives an overview of work related to the effects of $\mathrm{CO}_{2}$ on the human body. In the following we use this survey as a base for our selection and analysis of papers being relevant for our prototype. A reduction of cognitive performance scores could be measured in a controlled study by Satish et al. [33] with a $\mathrm{CO}_{2}$ concentration of $1000 \mathrm{ppm}$ after 2.5 hours. A reduction was observed by Allen et al. [12] in their controlled study with a $\mathrm{CO}_{2}$ concentration of about $950 \mathrm{ppm}$. Maula et al. [26] reported a slightly reduced cognitive performance of their controlled study participants at a $\mathrm{CO}_{2}$ concentration of $2260 \mathrm{ppm}$ after four hours. The perceived workload and fatigue was increased. However, they could not find a strong effect of human bioeffluents on the work performance.

Zhang et al. [36] could not observe a significant reduction of cognitive performance with increased $\mathrm{CO}_{2}$ concentrations of up to $3000 \mathrm{ppm}$ during their 4.25 hours long controlled experiments. Nevertheless, if bioeffluents were also present at $\mathrm{CO}_{2}$ concentrations of up to $3000 \mathrm{ppm}$ the cognitive performance was reduced. Additionally, their study participants reported the air quality as being lower and that they experienced increased intensity of headache, fatigue, sleepiness and difficulty in thinking. Zhang et al. supposed that they might not have been able to measure a dependency between cognitive performance and $\mathrm{CO}_{2}$ concentration due to their simpler cognitive tests in comparison to the strategic management simulation test that was for example used by Allen et al. [12] and Satish et al. [33].

In a more recent study by Allen et al. [11] investigated the performance of commercial airline pilots during a simulated three hours long flight with varied $\mathrm{CO}_{2}$ concentrations. Especially at a concentration of $2500 \mathrm{ppm}$ the performance of the pilots was significantly lower compared to $700 \mathrm{ppm}$. At $1500 \mathrm{ppm}$ a minor performance reduction could be observed.

Ratings of $\mathrm{CO}_{2}$ concentrations can also be found in official documents released by governments. For example in a proclamation of the German "Umweltbundesamt" [10], which is based on several studies before 2008, a $\mathrm{CO}_{2}$ concentration below $1000 \mathrm{ppm}$ is considered as inconspicuous, between $1000 \mathrm{ppm}$ and $2000 \mathrm{ppm}$ as conspicuous and above $2000 \mathrm{ppm}$ as unacceptable. Comparable guidelines are released by other countries' governmental institutions.

The results of our literature review shows no exact thresholds for our system. Nevertheless, the recommendations by the German "Umweltbundesamt" from 2008 are still in line with more recent literature. Therefore, we use these thresholds for our system: $1000 \mathrm{ppm}$ as bad and $2000 \mathrm{ppm}$ as very bad air quality. 


\subsection{Systems Monitoring Indoor Air Quality}

Marques et al. presented in [22] the "iAirCO ${ }_{2}$ " system which monitors indoor air quality. It uses an ESP8266 microcontroller with WiFi connectivity interfacing a NDIR-based (Nondispersive infrared sensor) $\mathrm{CO}_{2}$ sensor. The data can be accessed via an iOS smartphone app and a web interface. It can inform the user about bad air quality via e-mail, SMS or smartphone notifications. With our developed prototype we use also NDIR-based $\mathrm{CO}_{2}$ sensors and ESP8266 controllers are used to control the LED stripes wirelessly. For the system output we focus on unobtrusive notifications provided by ambient light that is observable by all persons inside the room. In our case there is no automatic ventilation available and no (external) person is responsible to sufficiently air the room so that the room occupants should be enabled by our system to air the room by themselves. Additionally, we try to prevent open windows to be forgotten when everybody leaves the room.

In the article [30] by Perez et al. an indoor environmental quality monitoring system for schools is being described which has been evaluated in five rooms. The $\mathrm{CO}_{2}$ concentration determined by a novel NDIR-based sensor, humidity and temperature of a sensor node were transmitted to a gateway via the Z-Wave wireless protocol. The observed rooms didn't provide automated heating, ventilation or air conditioning. The system was used to collect and analyze data and provides estimations how long a room should be aired. The authors included the humidity and temperature as these also affect human wellbeing. They conclude that it is necessary to use air monitors to be able to sufficiently air rooms at a school. Their system could be a low-cost solution for this problem. In this study the author's system did not directly notify the students or teachers or let them access the data. Thus, particularly the notification component of our developed system could be an addition to their sensor system. In comparison to Perez et al. we didn't take temperature and humidity into account as they are more likely to be noticed and adapted by airing or controlling the heating. Nevertheless, for a future prototype this might be a possible addition where also energy efficient airing could be considered.

A system intended to prevent sudden infant death which additionally to other data observes the indoor air quality is presented by De La Iglesia et al. in [18]. To observe several gas concentrations multiple low-cost gas sensors based on the MOS (Metal Oxide Semiconductor) principle are used which are interfaced by an ESP8266 microcontroller. In comparison to NDIR-based sensors the readings of MOS sensors usually include the gas concentrations of other gases which should be considered when interpreting the data. For this reason we use NDIR-based $\mathrm{CO}_{2}$ sensors for our system which are less influenced by other gases. To measure VOCs a combined value of several gases is intended and thus for this purpose MOS-based sensors are usually the better choice. For notifications rules are used that determine different severity levels of a problematic situation depended on the context. A mobile app is included to notify parents and caregivers. For emergency situations ambient lights are not intended to be used as they are more likely to be overlooked, nevertheless they can usually easily be switched 
to act obtrusively. In our case increased brightness or eye-catching brightness patterns like fast flashing would be possible. Nevertheless, the meaning of the encoding would have to be made clear.

A commercial device to monitor the air quality at home can be found with the "idevices" "Air Quality Sensor" [1]. It measures VOCs, $\mathrm{CO}_{2}$, temperature, humidity and air pressure and is connected to the company's cloud service where it stores the data. The product most likely incorporates the Bosch BME680 which is MOS-based using the compact MEMS fabrication (Micro-Electro-Mechanical System). The cloud service of "idevices" provides a web interface and smartphone app to access the sensor data. It can be integrated in several smart home systems. The sensor itself incorporates LEDs to indicate very good, alarming, and bad air quality. This commonly used traffic light color encoding is also applied to our system. The device itself does not provide hints what purpose it has so that the color of the LEDs is not directly understandable. We try to reduce this mapping problem by locating the ambient lights next to the windows so that there is a tight association with airing and windows status. As we use several LED stripes at least one of them is usually in the field of sight of persons inside the room so that it is very likely that at least one occupant notices the ambient notifications. All data of our system is stored inside the same room so that a high level of control and privacy can be guaranteed.

\subsection{Guidelines and Categorization of Ambient Light Systems}

For ambient information systems several guidelines and classifications can be found. Nevertheless, there are just few for ambient light systems which are explicitly dedicated for the modality "light".

In the work [25] by Matviienko et al. nine guidelines derived from their experimental results are listed. For our system we used the traffic light color encoding due to their guideline "GL4". This color mapping was found as being most frequently proposed by their study participants for everyday situations. According to traffic lights and "GL1" red is a negative and green a positive indicator.

Matviienko et al. additionally proposed a categorization of ambient lights in [24] and applied it to 72 ambient light systems. Their categorization is derived from several guidelines and heuristics for ambient information systems that can be found in the literature.

In the following we classify our system according to their work. Terms used by Matviienko et al. to classify systems are written in italics. Our system is showing the current status. Notifications which could distract the users are not implemented in our system since the provided information is never critical. Thus, we use color changes and just a slowly pulsed light pattern. The information of our system is encoded by the color (air quality), brightness pattern (window state) and LED position (right beside window handles / door). In the office is the context where our system is being used. The information sensitivity is public since several persons inside the room can see the LEDs. From the technical perspective we use multiple LEDs which can provide multicolor light. Our system is stationary. 


\subsection{Ambient Light in the Office Environment}

Ambient light can be used in several contexts. In this case we focus on the office context. Nevertheless, due to the usage of our system in a meeting room there are some identical requirements to be met which occur in the home context. For example, it is required to dim down ambient lights in low light conditions so that they don't become disturbing. In the home environment this can occur in the evening and night while the inhabitants are watching TV if an ambient light is placed nearby. This problem for example had to be dealt with in the work by Seiderer et al. [34] and could be observed for an ambient screen by Consolvo and Towle in [14]. The same issue occurs in the meeting room during presentations using the projector in the evening or when the room has to be darkened with the indoor and / or outdoor shutters. This is why we include an adaptation of the LEDs' brightness according to the brightness measured in the room.

Health related ambient light systems, trying to increase the amount of movement of individual office workers, were for example presented by Fortmann et al. [19] and Mateevitsi et al. [23]. The light sources were installed nearby the workplaces. To provide biofeedback with ambient light has also been researched in the work by $\mathrm{Yu}$ et al. [35] where it is used for stress intervention and relaxation. Two works by Müller et al. use ambient lights at the back of the PC monitor of workers. In the first work it is applied to remind them about upcoming events and appointments [28], in the second to let them keep track of secondary tasks in general [27]. The mentioned systems for individuals could be an addition to our system and can also improve the wellbeing of the workers or their time planning.

An ambient light system used in a meeting room to support the time management has been researched by Occhialini et al. [29]. Additionally, other systems can be found that are intended to provide unobtrusive information during presentations so that the course of a meeting can be adapted accordingly. Our system should not intervene with the meetings and just provide feedback so that the room can be aired adequately by the persons preventing a reduction of wellbeing and cognitive performance of the participants due to bad air quality.

\section{Implementation}

\subsection{Hardware}

Figure 1 shows an overview of the hardware that we employed. A Raspberry Pi $3 \mathrm{~B}+$ connected via WiFi served as the central device that runs all the software that is necessary to acquire, process and store sensor data and to control LED actuators based on rules. Our prototype included two different low budget $\mathrm{CO}_{2}$ sensors and a VOCs sensor in order to compare their readings, their dependency on temperature, humidity, and air pressure and observe general sensor drifts. A final setup requires just one reliable sensor.

Figure 1 further shows that an Arduino Mini Pro is connected to the Raspberry. It is supplied with $3.3 \mathrm{~V}$ at $8 \mathrm{MHz}$ and interfaces via $\mathrm{I}^{2} \mathrm{C}$ a Sensirion 


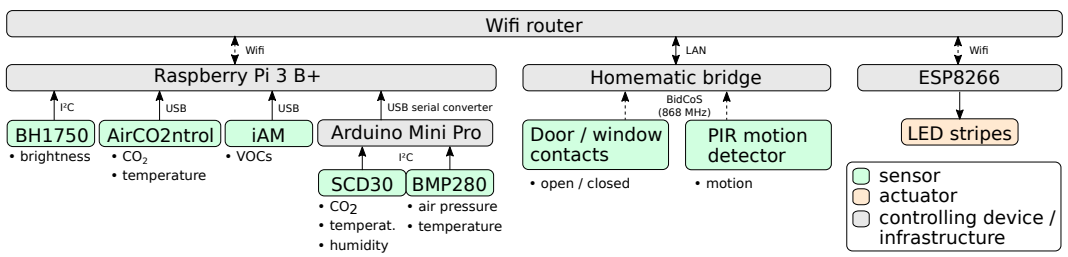

Fig. 1. Hardware overview of the prototype.

SCD30 [2] NDIR based calibrated $\mathrm{CO}_{2}$ sensor and a Bosch BMP280 [3] air pressure sensor. The SCD30 incorporates a temperature and humidity sensor which allows the sensor's firmware to compensate the dependencies on temperature and humidity. An additional compensation on air pressure is possible for which the BMP280 sensor would be used. The Arduino, SCD30 and BMP280 components are enclosed in a $3 \mathrm{~d}$ printed case with airing slots.

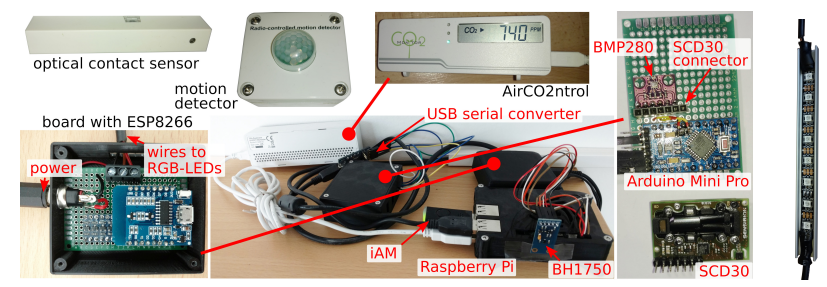

Fig. 2. The picture shows the Raspberry Pi 3 B+ with the gas, temperature, humidity, air pressure, light sensors located next to the door on a sideboard. Additionally, one of the three identical ESP8266 boards for the RGB-LED stripes, the Homematic motion detector and one of the six Homematic optical contact sensor are visible. On the right one of the six LED-stripes with its eight LEDs glued to an aluminum profile is shown without the translucent shade.

A TFA Dostmann AirCO2ntrol Mini [4] $\mathrm{CO}_{2}$ sensor, which includes a temperature reading, is connected to the Raspberry $\mathrm{Pi}$ via USB. This sensor is built as a consumer device and more cost-effective $(\sim 40 €)$ than the more recent SCD30 $(\sim 60 €)$. We placed the sensor so that the display and the LEDs are not visible to room occupants. According to data sheets, the SCD30 is able to measure $\mathrm{CO}_{2}$ concentrations in a range of 400 to $10,000 \mathrm{ppm}$ (calibrated) while the AirCO2ntrol sensor is capable of a range of 0 to $3000 \mathrm{ppm}$.

Furthermore, an ams iAM [5] VOCs sensor is connected via USB to the Raspberry Pi. As typical for VOCs sensors, it delivers values in ppm which are relative to the air quality at power-up or a previously stored value.

To get information about the status of windows and door, and movement in the room, we employ battery powered sensors of the Homematic [6] wireless system. At each window and door, an optical contact sensor (HM-SEC-SCo) is placed. One motion sensor (HM-SEN-MDIR-SM) is located at the side of 
the room. The sensor data can be received by means of a bridge device that is connected to the router via LAN.

The ambient brightness in lux in the room is determined by a BH1750 brightness sensor connected to the Raspberry Pi via $\mathrm{I}^{2} \mathrm{C}$.

As actuators, we are using six (door: 1, windows: 5) RGB-LED stripes consisting out of eight WS2812B LEDs (60 LEDs per meter) each which are controlled via three ESP8266 microcontrollers. Each stripe is encased in an aluminum profile with a translucent plastic shade. Since high currents are required if the LEDs are running with white color and full brightness (max. $60 \mathrm{~mA}$ per LED), $5 \mathrm{~V}$ power supplies with $2.5 \mathrm{~A}$ maximum current are used to prevent unexpected behavior of the controllers or LEDs due to power breakdowns. A maximum of three stripes (24 LEDs) is connected to one ESP8266 and power supply. The microcontrollers are connected to the WiFi. The ESP8266 board with connectors and a level shifter $(5 \mathrm{~V} \leftrightarrow 3.3 \mathrm{~V})$ are protected by a $3 \mathrm{~d}$ printed case.

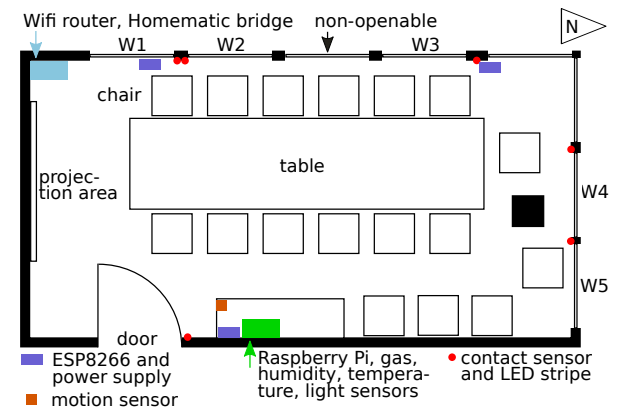

Fig. 3. Overview of the meeting room with hardware positions. Openable windows are labeled with $\mathrm{Wx}$ where $\mathrm{x}$ is a number. Positions and lengths in general and the count of chairs / non-openable windows are not exact.

\subsection{Software}

An overview of the communication between the software is visible in Figure 4 .

On the Raspberry Pi Raspbian Linux is installed. For communication between some software components the Mosquitto MQTT broker is used. For data storage and partly also processing PostgreSQL is set up. The lightweight opensource home automation software FHEM [7] interfaces the Homematic sensors, reads out the sensor data of the iAM USB stick, acquires the brightness data of the BH1750 and receives all other sensor data via MQTT. Additionally, it is connected with the software on the ESP8266 controllers to be able to control them. We adapted a code to read out the sensor data of the AirCO2ntrol device so that it can transmit data via MQTT. It is shared on Github ${ }^{1}$. Grafana is used to visualize and explore data in the database in real time.

\footnotetext{
${ }^{1}$ https://github.com/andreas-seiderer/co2mon
} 
To receive data from the Arduino Mini Pro (for event processing and context interpretation), we use our own open-source software called Eventerpretor ${ }^{2}$ which was already used in [32] and has been extended since then. Our software is written in Kotlin (instead of native or hybrid frameworks, e.g., [15]), can be used on Windows and Linux, and improves and extends the functionality of the single threaded FHEM for this project. It additionally allows replacing FHEM or including extensions without the need of major changes of the rules. This is especially beneficial for prototyping. A list of benefits in comparison to FHEM relevant for this project is shown in the following:

- flow-based programming with several reusable nodes,

- multi-threaded processing (the Raspberry Pi's 4 CPU cores can be utilized),

- database provided data aggregations,

- independent sensor model and rule engine (Drools),

- caching / synchronization of the rule engine's facts,

- custom web interfaces and replay function of recorded events for testing,

- generic serial port interfacing.

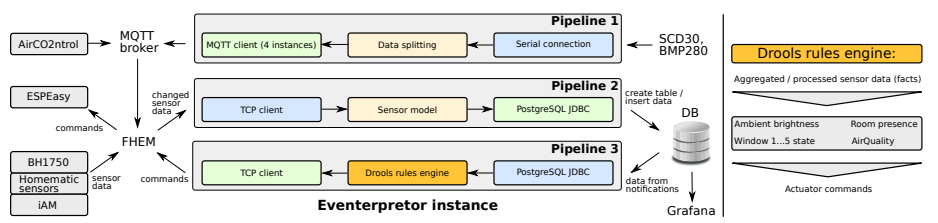

Fig. 4. Software processing and communication overview.

Some functionality is comparable to the more complex Node-RED by IBM for Node.js, nevertheless Eventerpretor includes for our purposes required missing features. Due to the use of Java, we can use real multi-threading for each node and integrate any Java library.

Three pipelines are running in one instance of Eventerpretor. They are visible in Figure 4. The first pipeline receives data of the Arduino Mini Pro from the serial port and processes them (e.g. value separation) so that they can be sent via MQTT to FHEM.

The second pipeline directly receives all sensor events of FHEM. FHEM is configured in a way that only transitions of different values are processed as new events. The events of FHEM are parsed by Eventerpretor and supplemented with metadata such as sensor type or sensor position. This additional data is used to automatically create tables with sensor data in the database and to add new sensor entries in a "sensors" table where the additional sensor information can be accessed and the related tables are referenced. Since Eventerpretor is implementing its own sensor model, it is possible to use any other program that can interface Homematic devices and the iAM sensor.

\footnotetext{
$\overline{2}$ https://github.com/andreas-seiderer/Eventerpretor
} 
The third pipeline receives processed sensor data from the database. This is implemented in a way that it creates trigger functions that can aggregate sensor data over time. For example, the average sensor value over the last five minutes can be calculated whenever a new sensor value has been inserted by the second pipeline. After the calculation, a notification is sent to all subscribers including the calculated value - in this case Eventerpretor listens to the trigger that it has created. The possibility of asynchronous messaging is a special function of PostgreSQL and can be very useful to prevent inefficient polling loops. After receiving the aggregated values, the pipeline uses the data with the sensor model (facts) with the rule engine "Drools" [8]. The rule system first generates context elements like the interpretation of the air quality out of the $\mathrm{CO}_{2}$ concentration and finally creates the commands (cf. right side of Figure 4). The commands are fed to FHEM which controls the different RGB-LED stripes. Just new commands are sent to prevent a high CPU load of the ESP8266 controllers.

Eventerpretor can directly host websites that allow simulating sensor data via a websocket connection but also can be used to receive and visualize events. This is useful during the development and prototyping process. Additionally, received events can be recorded and replayed to simulate specific situations like entering the room and opening a window with realistic sensor data and timing.

The air pressure is acquired by the Arduino Mini Pro firmware from the BMP280 sensor and directly sent to the SCD30 firmware for data compensation. After receiving the data from the SCD30 the Arduino sends all its readouts $\left(\mathrm{CO}_{2}\right.$ in ppm, temperature in ${ }^{\circ} \mathrm{C}$, humidity in \%) and BMP280 (air pressure in $\mathrm{hPa}$ and temperature in ${ }^{\circ} \mathrm{C}$ ) to the Raspberry Pi via a USB serial converter.

The ESP8266 boards are flashed with ESPEasy [9]. This firmware allows an easy setup of the controller via a webinterface. It can be used to connect it to a password protected WiFi without having to hard code the SSID and password. Additionally, it can interface sensors but also actuators. In our case we set up the WS2812B LED stripes which then can be controlled over the network. Since we required new brightness patterns we created a new plugin for ESPEasy.

\subsection{Setup}

In Figure 3 the positions of the hardware are visible. In Figure 5 pictures of LED-stripes next to the door a) and four of the five windows b) and c) are visible. The room has a volume of about $93.4 \mathrm{~m}^{3}$ and is used by less than 20 persons at the same time. Through W1, W2 and W3 and non-openable windows it is possible that direct sunlight can enter the room. W3 and W4 are directed to the north.

If the projector is used and there is too much sunlight shining into the room, indoor and electrical outdoor shutters can be closed. The indoor shutters can manually be moved with a cord horizontally and the vertical angle of the shutter's stripes can be adapted, too. These shutters are located in front of the LED stripes so that they are no longer in sight if they are completely closed. In this case just the LED stripe at the door is directly visible. 

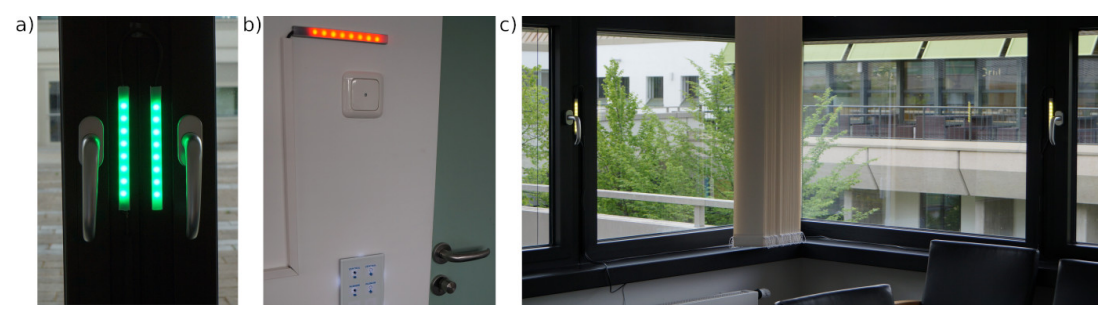

Fig. 5. a) shows the LED-stripes next to window W1 and W2. In b) the stripe next to the door can be seen. In c) the stripes next to window W3 and W4 are visible. Additionally, two non-openable windows and one of the manual indoor shutters can be noticed. For demonstration purposes all three colors are shown in the pictures.

The Raspberry Pi including the gas sensors is placed on a sideboard which is about $110 \mathrm{~cm}$ high so that the $\mathrm{CO}_{2}$ concentration is measured at about the usual height of sitting persons. The room has five windows that can be opened and one door which leads into a corridor. At the side of each window handle a LED stripe is placed. At each window and door a contact sensor is added.

\section{Evaluation}

For a basic evaluation of ambient displays the heuristics by Mankoff et al. [21] can be used to identify major problems already during the development process. Three instructed persons applied the heuristics on our system. As a result we changed the pulsed brightness pattern so that it doesn't completely dim down to dark but to a lower brightness since it was considered as being too obtrusive.

As a first step, we used the sensors to record data without providing any notifications. This phase of the system was used to test and improve the stability of the prototype. Additionally, the recorded data shows what gas concentrations are to be expected in this room, how often it is being aired and how the different gas sensors behave. After that we activated the notifications and conducted a workshop to gather further insights about the system.

\subsection{Data recording}

First data recordings were conducted during winter and spring 2018 / 2019. The system is intended to gather long-term data to allow more detailed analysis in the future. Figure 6 shows example data which we discuss in more detail.

The visible data of the meeting room was recorded in February where a maximum outside temperature of $-1.6^{\circ} \mathrm{C}$ was reached. At the top the gas concentrations of $\mathrm{CO}_{2}$ and VOCs are visible provided by the three gas sensors. Below the temperature readings of the AirCO2ntrol sensor are shown. After that the state of the door and the five windows (W1 - W5) is plotted.

The $\mathrm{CO}_{2}$ readings of the AirCO2ntrol and SCD30 sensors just slightly differ. The AirCO2ntrol data shows in general a more noisy signal. At the selected 


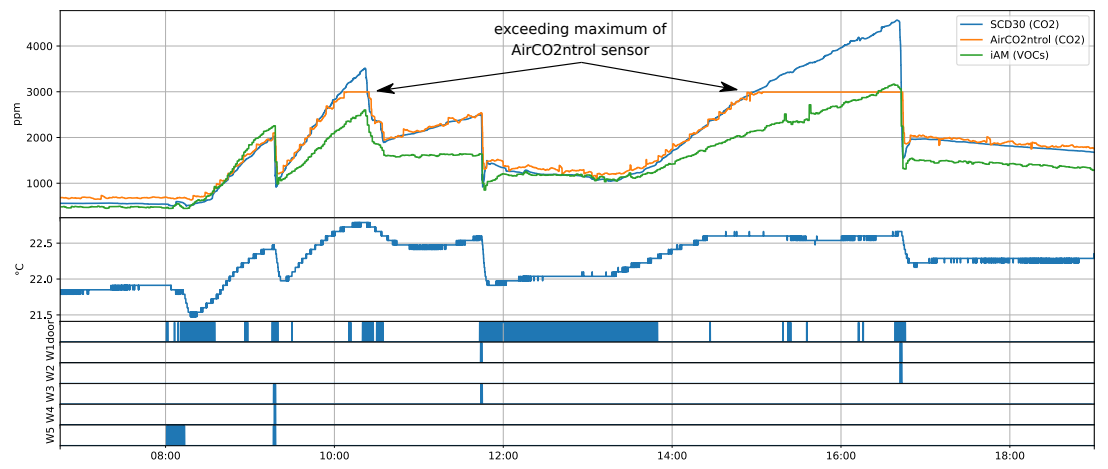

Fig. 6. Example gas sensor measurements in the meeting room of a day in February 2019. The VOCs (related to "fresh" air) and $\mathrm{CO}_{2}$ (factory calibration) concentrations can just be compared by their relative value changes. In this case no higher concentrations of gases next to $\mathrm{CO}_{2}$ could be detected by the iAM sensor (this would have resulted in peaks only shown by this sensor). The maximum outside temperature was around $-1.6^{\circ} \mathrm{C}$. Drop downs in $\mathrm{CO}_{2}$ and VOCs concentrations indicate an open door or open windows (W1 - W5). The $\mathrm{CO}_{2}$ measurement range of the AirCO2ntrol sensor was exceeded two times.

day the maximum range of the sensor has been exceeded two times so that for this room it is necessary to use sensors with a higher maximum range like the SCD30 sensor to be able to observe the maximum $\mathrm{CO}_{2}$ concentrations. The VOCs concentration is strongly depended on the $\mathrm{CO}_{2}$ concentration and in this case shows similar curve shapes. Nevertheless, this is not always true and in such cases indicates the presence of other gases. Although the VOCs sensor is just roughly calibrated it could be useful for our system to be able to notify about other potentially unpleasant gases in the room air.

The temperature measured by the AirCO2ntrol sensor didn't change much during the day (in a range of about $1.5{ }^{\circ} \mathrm{C}$ ). The gas concentration values and the temperature responded quite quickly to open windows. As soon as just the door was open, the speed of the gas concentration drop is lower.

In general, it can be seen that the room was aired multiple times but not often and partly not long enough so that relatively high gas concentrations could be measured inside the room. One reason for too short airing might have been the lower temperatures outside but more likely the air was no longer noticed as being bad. Especially during the long person presence around 14 o'clock the room occupants did not recognize the bad air quality and the room should have been aired. Although the door was partly opened for longer time periods, this just slightly reduced the VOCs and $\mathrm{CO}_{2}$ concentrations or caused them to stay at a relatively high level.

During the total recorded time period in winter and spring there are several days when bad and very bad air quality was reached inside the room but very rarely with gas concentrations above $3000 \mathrm{ppm}$. Thus, with activated ambient display our system has high potential in improving this situation. 


\subsection{Workshop with Meeting Room Users}

In order to gain insights from staff members who have been using the meeting room on a regular basis we conducted a workshop. Workshop participants were five staff members with expertise in human-robot interaction, machine learning, or mobile applications. Three researchers conducted the workshop. One researcher facilitated the workshop and two additional researchers took notes. The workshop took place in the meeting room where the system was installed. Initially, the five participants were given a brief description to the system in room, including a reminder/demonstration of how the system's ambient feedback changes when the air quality gets worse and when it gets better.

After that, the facilitator guided a semi-structured discussion. It aimed at identifying limitations, potentials, and general attitudes of the participants regarding the interaction design. To ease the discussion, it was structured into (i) collecting feedback on potentials and weaknesses associated with the prototype system, (ii) additional features participant missed and would desire in a future system, (iii) use cases of how such an ambient display could be used in other application domains than a meeting room, and (iv) an open discussion on anything that we may have missed asking for. The workshop took exactly one hour.

\subsection{Workshop Results and Discussion}

Overall, four participants stated that they would want to have such a system installed in their offices. Only one participant stated that they had already established a daily routine of opening the office windows, which they believed fulfilled their needs. Most of the participants comments addressed potentials of the system. The main weakness and limitation of the system was identified as usability issues, but only if one would use the ambient lights to display more and diverse information. For example displaying a history of air quality for a single room or use animation on LED stripes to indicate bad air quality in another room.

The main insights gained from the workshop are centered around using technology for ambient interaction to enabling a new form of "ambient materiality ", which grants users more agency associated with air quality in future urban spaces, which will be discussed hereafter.

Towards Interacting with "Ambient Materiality" We summarize a set of comments made by the participants with the topic "Ambient Materiality", because comments highlighted how the ambient and located light sources provided "material qualities" to air, which is usually invisible, non-graspable and nearly immaterial. Participants argued, for example, how by placing the LED stripes in different locations in the room (or an apartment) one might get a system being able to make recommendations on which windows to open and for how long to get fresh air to desired spaces (and rooms). Furthermore, participants highlighted that notifications on their mobiles would be very desirable and helpful in case one forgets to close a window or to receive reminders considering air qualities in other/remote rooms. One participant provided as an example, how 
they don't open the windows in their bedroom at home because they usually forget to close the windows and end up freezing at nighttime. They argued that a reminder to close the windows once the room has established good air quality would be very much appreciated. However, participants also argued how there has to be balance between air quality, room temperature, humidity, and pollen. Consequently, there was a discussion about potential sensors also placed outside the windows to get recommendations on when to open windows to get good air quality without making it accidentally worse.

Digital Air Displays in Urban Spaces A second topic that was discussed dealt with how digital air quality displays could transform various (urban) spaces. Workshop participants mentioned for example, how displays on public transportation could be useful in deciding which route or vehicle to choose for transportation. One participant argued that they didn't see a concrete benefit of having air quality displays in public spaces other than to sensibilize fellow townsmen. One participant argued that there are already temperature displays which they appreciate without concrete benefit. Participants also discussed the potential of standardizing air quality sensors/displays on windows to monitor and regulate air quality in future digital cities.

In sum, the workshop has demonstrated that an ambient air quality display is very desirable and not only for meeting rooms but in general for urban regions. The participants' discussions have shed light on the diverse potential of ambient air quality displays and provided many ideas and inspirations for follow research and development work.

\section{Conclusion}

We presented the design and implementation of an ambient light notification system that unobtrusively informs people about the indoor air quality with the aim of improving it when necessary. Long-term measurements of our evaluation (without activated ambient lights) showed that the indoor air often became very poor without the users ventilating the room. Also, the ventilation phases often did not last long enough to improve the indoor air sufficiently. This part of the evaluation showed that an optimization of the ventilation behavior would be necessary or at least meaningful and effective. A workshop in the room (with activated ambient lights) showed that people understood the ambient notifications and would potentially lead to better indoor air quality. The workshop further revealed many more use cases such as including room temperature and humidity, weather, and outdoor air quality data into the semantics of ambient notifications. Even harmful gases outside the room, e.g., nitrogen dioxide or fine dust such as used in [17], may be considered as warnings in the ambient notifications in order to optimize ventilation phases. Additionally, our prototype could be researched in AAL settings by adding it to recommender systems like presented in [31].

Overall, we have shown that our system has the potential to improve indoor air quality and to create a healthier environment. 


\section{Acknowledgments}

We want to thank Andrés Caro Quintul for his help with parts of the prototype.

\section{References}

1. https://www.idevices.de/english/

2. https://www.sensirion.com/de/umweltsensoren/kohlendioxidsensoren-co2/

3. https://www.bosch-sensortec.com/bst/products/all_products/bmp280

4. https://www.tfa-dostmann.de/en/produkt/co2-monitor-airco2ntrol-mini/

5. https://ams.com/iam

6. https://www.eq-3.com/products/homematic.html

7. https://www.fhem.de/

8. https://www.drools.org/

9. https://www.letscontrolit.com/wiki/index.php?title=ESPEasy

10. -: Gesundheitliche Bewertung von Kohlendioxid in der Innenraumluft. Bundesgesundheitsblatt - Gesundheitsforschung - Gesundheitsschutz 51(11) (Nov 2008)

11. Allen, J.G., MacNaughton, P., Cedeno-Laurent, J.G., Cao, X., Flanigan, S., Vallarino, J., Rueda, F., Donnelly-McLay, D., Spengler, J.D.: Airplane pilot flight performance on 21 maneuvers in a flight simulator under varying carbon dioxide concentrations. Journal of exposure science \& environmental epidemiology (2018)

12. Allen, J.G., MacNaughton, P., Satish, U., Santanam, S., Vallarino, J., Spengler, J.D.: Associations of Cognitive Function Scores with Carbon Dioxide, Ventilation, and Volatile Organic Compound Exposures in Office Workers: A Controlled Exposure Study of Green and Conventional Office Environments 124(6), 805 (2016)

13. Azuma, K., Kagi, N., Yanagi, U., Osawa, H.: Effects of low-level inhalation exposure to carbon dioxide in indoor environments: A short review on human health and psychomotor performance. Environment International 121, 51 - 56 (2018)

14. Consolvo, S., Towle, J.: Evaluating an ambient display for the home. In: CHI'05 extended abstracts. pp. 1304-1307. ACM (2005)

15. Dang, C.T., André, E.: A Framework for the Development of Multi-display Environment Applications Supporting Interactive Real-time Portals. pp. 45-54. EICS '14, ACM, New York, NY, USA (2014). https://doi.org/10.1145/2607023.2607038

16. Dang, C.T., Andre, E.: Acceptance of Autonomy and Cloud in the Smart Home and Concerns. In: Dachselt, R., Weber, G. (eds.) Mensch und Computer 2018 Tagungsband. Gesellschaft fuer Informatik e.V., Bonn (2018)

17. Dang, C.T., Seiderer, A., André, E.: Theodor: A Step Towards Smart Home Applications with Electronic Noses. In: Proceedings of the 5th International Workshop on Sensor-based Activity Recognition and Interaction. pp. 11:1-11:7. iWOAR '18, ACM, New York, NY, USA (2018)

18. De La Iglesia, D.H., De Paz, J.F., Villarrubia González, G., Barriuso, A.L., Bajo, J.: A Context-Aware Indoor Air Quality System for Sudden Infant Death Syndrome Prevention. Sensors 18(3) (2018)

19. Fortmann, J., Stratmann, T.C., Boll, S., Poppinga, B., Heuten, W.: Make me move at work! an ambient light display to increase physical activity. In: Proceedings of the 7 th international conference on pervasive computing technologies for healthcare. pp. 274-277 (2013)

20. Gunnarsen, L., Fanger, P.O.: Adaptation to indoor air pollution. Environment International 18(1), $43-54$ (1992) 
21. Mankoff, J., Dey, A.K., Hsieh, G., Kientz, J., Lederer, S., Ames, M.: Heuristic evaluation of ambient displays. In: Proceedings of the SIGCHI conference on Human factors in computing systems. pp. 169-176. ACM (2003)

22. Marques, G., Ferreira, C.R., Pitarma, R.: Indoor Air Quality Assessment Using a CO 2 Monitoring System Based on Internet of Things. Journal of medical systems 43(3), 67 (2019)

23. Mateevitsi, V., Reda, K., Leigh, J., Johnson, A.: The health bar: a persuasive ambient display to improve the office worker's well being. In: Proceedings of the 5th augmented human international conference. ACM (2014)

24. Matviienko, A., Rauschenberger, M., Cobus, V., Timmermann, J., Fortmann, J., Löcken, A., Müller, H., Trappe, C., Heuten, W., Boll, S.: Towards new ambient light systems: a close look at existing encodings of ambient light systems. Interaction Design and Architecture (s). 2015;(26): 10-24. (2015)

25. Matviienko, A., Rauschenberger, M., Cobus, V., Timmermann, J., Müller, H., Fortmann, J., Löcken, A., Trappe, C., Heuten, W., Boll, S.: Deriving design guidelines for ambient light systems. In: Proceedings of the 14th international conference on mobile and ubiquitous multimedia. pp. 267-277. ACM (2015)

26. Maula, H., Hongisto, V., Naatula, V., Haapakangas, A., Koskela, H.: The effect of low ventilation rate with elevated bioeffluent concentration on work performance, perceived indoor air quality, and health symptoms. Indoor air 27(6) (2017)

27. Müller, H., Kazakova, A., Heuten, W., Boll, S.: Lighten Up!-An Ambient Light Progress Bar Using Individually Controllable LEDs. In: European Conference on Ambient Intelligence. pp. 109-124. Springer (2015)

28. Müller, H., Kazakova, A., Pielot, M., Heuten, W., Boll, S.: Ambient timerunobtrusively reminding users of upcoming tasks with ambient light. In: IFIP Conference on Human-Computer Interaction. Springer (2013)

29. Occhialini, V., Van Essen, H., Eggen, B.: Design and evaluation of an ambient display to support time management during meetings. In: IFIP Conference on Human-Computer Interaction. pp. 263-280. Springer (2011)

30. Ortiz Perez, A., Bierer, B., Scholz, L., Wöllenstein, J., Palzer, S.: A wireless gas sensor network to monitor indoor environmental quality in schools. Sensors 18(12)

31. Rist, T., Seiderer, A., André, E.: Providing Life-Style-Intervention to Improve Well-Being of Elderly People. In: Entertainment Computing - ICEC 2018. pp. 362-367. Springer International Publishing, Cham (2018)

32. Ritschel, H., Seiderer, A., Janowski, K., Aslan, I., André, E.: Drink-O-Mender: An Adaptive Robotic Drink Adviser. In: Proceedings of the 3rd International Workshop on Multisensory Approaches to Human-Food Interaction. pp. 3:1-3:8. MHFI'18, ACM, New York, NY, USA (2018)

33. Satish, U., Mendell, M.J., Shekhar, K., Hotchi, T., Sullivan, D., Streufert, S., Fisk, W.J.: Is CO2 an indoor pollutant? Direct effects of low-to-moderate CO2 concentrations on human decision-making performance. Environmental health perspectives 120(12), 1671 (2012)

34. Seiderer, A., Dang, C.T., André, E.: Exploring Opportunistic Ambient Notifications in the Smart Home to Enhance Quality of Live. In: Enhanced Quality of Life and Smart Living. pp. 151-160. Springer International Publishing, Cham (2017)

35. Yu, B., Hu, J., Funk, M., Feijs, L.: Delight: biofeedback through ambient light for stress intervention and relaxation assistance. Personal and Ubiquitous Computing 22(4), 787-805 (2018)

36. Zhang, X., Wargocki, P., Lian, Z., Thyregod, C.: Effects of exposure to carbon dioxide and bioeffluents on perceived air quality, self-assessed acute health symptoms, and cognitive performance. Indoor air 27(1), 47-64 (2017) 